\title{
BMJ Open Risk of early neurodevelopmental outcomes associated with prenatal exposure to the antiepileptic drugs most commonly used during pregnancy: a French nationwide population-based cohort study
}

\author{
Pierre-Olivier Blotière (D) , ${ }^{1,2}$ Sara Miranda, ${ }^{3}$ Alain Weill, ${ }^{1}$ Yann Mikaeloff, ${ }^{4,5}$ \\ Hugo Peyre, ${ }^{6,7,8}$ Franck Ramus, ${ }^{6}$ Zureik Mahmoud, ${ }^{3,9}$ Joël Coste, ${ }^{1,10}$ \\ Rosemary Dray-Spira (i) ${ }^{3}$
}

To cite: Blotière $\mathrm{P}-0$, Miranda $\mathrm{S}$, Weill A, et al. Risk of early neurodevelopmental outcomes associated with prenatal exposure to the antiepileptic drugs most commonly used during pregnancy: a French nationwide population-based cohort study. BMJ Open 2020;10:e034829. doi:10.1136/ bmjopen-2019-034829

- Prepublication history and additional material for this paper are available online. To view these files, please visit the journal online (http://dx.doi. org/10.1136/bmjopen-2019034829).

Parts of this work have been previously presented at the 34th International Conference on Pharmacoepidemiology and Therapeutic Risk Management in Prague, Czech Republic (August 2018).

Received 08 0ctober 2019 Revised 14 February 2020 Accepted 09 April 2020

Check for updates

(c) Author(s) (or their employer(s)) 2020. Re-use permitted under CC BY-NC. No commercial re-use. See rights and permissions. Published by BMJ.

For numbered affiliations see end of article.

Correspondence to Dr Pierre-Olivier Blotière; poblotiere@gmail.com

\section{ABSTRACT}

Objectives To assess the association between prenatal exposure to monotherapy with the antiepileptic drugs (AEDs) most commonly used during pregnancy and the risk of various neurodevelopmental outcomes compared with lamotrigine.

Design Nationwide population-based cohort study. Setting French national healthcare databases.

Participants Children born alive between 2011 and 2014 and prenatally exposed to AED monotherapy.

Primary and secondary outcome measures Outcomes included neurodevelopmental disorders (NDD), defined by International Classification of Diseases, 10th Revision codes F70-F98-pervasive developmental disorders (PDD, F84) and mental retardation (MR, F70-F79) were studied separately — and visits to speech therapists. The reference group comprised children prenatally exposed to lamotrigine. Children were followed until outcome, loss to follow-up, death or 31 December 2016. We performed inverse probability of treatment weighting analyses using the propensity score, which included maternal and infant characteristics. Hazard ratios (HRs) were calculated using Cox models.

Results The cohort comprised 9034 children, 2916 of which were exposed to lamotrigine, 1627 to pregabalin, 1246 to clonazepam, 991 to valproic acid (VPA), 621 to levetiracetam, 502 to carbamazepine, 477 to topiramate, 378 to gabapentin and 143 to oxcarbazepine. None of these AEDs, except VPA, was associated with an increased risk of any of the four neurodevelopmental outcomes investigated. Exposure to VPA was associated with increased risks of NDDs (HR=2.7, 95\% Cl (1.8 to 4.0)), PDD ( $\mathrm{HR}=4.4$ (2.1 to 9.3)), MR ( $\mathrm{HR}=3.1$ (1.5 to 6.2)) and visits to speech therapists $(\mathrm{HR}=1.5$ (1.1 to 1.9)), with a dose-response relationship.

Conclusions No increased risk of any of the neurodevelopmental outcomes investigated in this study was observed with prenatal exposure to levetiracetam, pregabalin, oxcarbazepine, topiramate, gabapentin, clonazepam or carbamazepine, compared with
Strengths and limitations of this study

- This nationwide cohort study based on the French healthcare databases is the largest study to date to assess the association between antiepileptic drug (AED) exposure during pregnancy and neurodevelopmental outcomes in the offspring.

- This study investigated a wide range of AEDs, including some drugs for which little or no information is available in the literature.

- Although residual confounding by unmeasured covariates cannot be excluded, the choice of lamotrigine as the reference group and the sensitivity analysis restricted to women considered to be treated for epilepsy should mitigate confounding.

- The maximum length of follow-up was 6 years, allowing only early diagnoses of neurodevelopmental disorders to be identified.

lamotrigine. However, this study corroborates the wellknown association between maternal use of VPA during pregnancy and the risk of neurodevelopmental disorders in the offspring. Longer follow-up is necessary to confirm these findings.

\section{INTRODUCTION}

Antiepileptic drugs (AEDs) are commonly prescribed during pregnancy to treat epilepsy and various other conditions, such as neuropathic pain syndromes, psychiatric disorders and chronic migraine ${ }^{1}$ : between $0.4 \%$ and $0.7 \%$ of women are exposed to AEDs during pregnancy in Europe ${ }^{23}$ while this prevalence is as high as $2 \%$ in the US. ${ }^{4}$

Some of these AEDs are known to be teratogens. ${ }^{5}$ Of all AEDs, prenatal exposure to valproic acid has been most clearly 
associated with poor neurodevelopmental outcomes, ${ }^{6}$ which led the US Food and Drug Administration to issue a warning in $2011^{7}$ and stringent guidance in 2013 for clinicians prescribing valproic acid to pregnant women or women of childbearing potential. ${ }^{8}$ The European Medicines Agency also strengthened warnings on the use of valproate-containing medicines in women and girls in $2014^{9}$ and issued a ban in 2018 on the use of such medicines during pregnancy for migraine or bipolar disorder, and for epilepsy except when no other effective treatment is available. ${ }^{10}$

Lamotrigine has generally been associated with favourable neurodevelopmental outcomes. ${ }^{6}$ Discordant but mainly reassuring data have been published for carbamazepine, while evidence for levetiracetam and topiramate remains limited and almost no information is available concerning the other AEDs, particularly clonazepam, oxcarbazepine, gabapentin and pregabalin. ${ }^{6}$ Furthermore, most of the studies conducted to date have been based on small sample sizes and may be prone to selection bias.

We therefore conducted a large-scale nationwide cohort study using the French healthcare databases to assess various early neurodevelopmental outcomes among children prenatally exposed to monotherapy with individual AEDs compared with lamotrigine-exposed children.

\section{METHODS}

\section{Study design and data sources}

The French national health insurance database (Système national d'information interrégimes de l'Assurance maladie (SNIIRAM)) and the French hospital discharge database (Programme de médicalisation des systèmes d'information (PMSI)) linked by a unique patient identifier were used to conduct this nationwide populationbased cohort study. ${ }^{11}$

The SNIIRAM database contains all individualised and anonymous healthcare claims reimbursed by French National Health Insurance, in particular all dispensed drugs and medical procedures in the outpatient setting. The SNIIRAM database also collects patient data such as age, gender, vital status and eligibility for complementary universal health insurance (CMU-C), which provides free access to healthcare for low-income people. ${ }^{12}$ Eligibility for $100 \%$ health insurance coverage for serious and costly long-term diseases (LTD) is also recorded in the SNIIRAM database.

The PMSI database provides detailed medical information on all admissions to public and private hospitals in France, including primary, related and associated discharge diagnoses, medical procedures and data related to pregnancy such as gestational ages and birth weights.

Drugs are coded according to the Anatomical Therapeutic Chemical (ATC) classification and medical procedures are coded according to the French medical classification of clinical procedures (Classification commune des actes médicaux (CCAM)). LTD and hospital discharge diagnoses are coded according to the International Classification of Diseases, 10th Revision (ICD-10).

This linkage has previously been used to conduct epidemiological studies in pregnancy research. ${ }^{313-15}$

\section{Study population}

All live births between January 2011 and December 2014 were eligible for inclusion. These live births were identified by using a published algorithm based on discharge diagnoses and medical procedures indicative of completion of pregnancy. ${ }^{3}$

The mother had to be enrolled in the national health insurance general scheme ( $75 \%$ of the French population), during the penultimate year before pregnancy. Pregnancies that could not be linked to neonatal data and twin pregnancies were excluded, as well as pregnancies for which the child had no valid identifier allowing follow-up and pregnancies for which gestational ages or birth weights were not available. Children with a hospital discharge diagnosis of brain malformation documented at birth were also excluded (see online supplementary table 1 for ICD-10 codes). For each woman, only the first birth occurring during the study period was considered.

Finally, only pregnancies exposed to AED monotherapy were included in the study population.

These exclusion criteria are reported in the study population flow chart (figure 1).

\section{Patient and public involvement}

Patients or the public were not involved in the design and the conduct of the study.

\section{Exposure}

All AEDs were studied (online supplementary table 2). Women were considered to be exposed during the 30 days following dispensing of an AED, as AED prescriptions are dispensed with a 30-day supply in France. Women were therefore exposed during pregnancy when an AED had been dispensed between 30 days before the beginning of pregnancy and the end of pregnancy. Monotherapy was defined as the absence of any other AED dispensed during the same period. Results related to AEDs rarely used as monotherapy during pregnancy $(<100$ exposed pregnancies) were not reported. The reference group included pregnant women exposed to lamotrigine monotherapy for the following reason: active-comparator designs minimise confounding by indication compared with the use of an unexposed control group ${ }^{16}$; lamotrigine is the most commonly used AED in France for the treatment of epilepsy; prenatal exposure to lamotrigine has been mostly shown to be associated with favourable neurodevelopmental outcomes; comparing all individual AEDs with lamotrigine addresses a clinically relevant question: which is the safest AED?

Mean daily doses were calculated by dividing cumulative doses by the number of days covered. Cumulative doses were assessed by equally distributing the dose of AED dispensed over the 30 days following dispensing 
Live births between January 2011 and December 2014
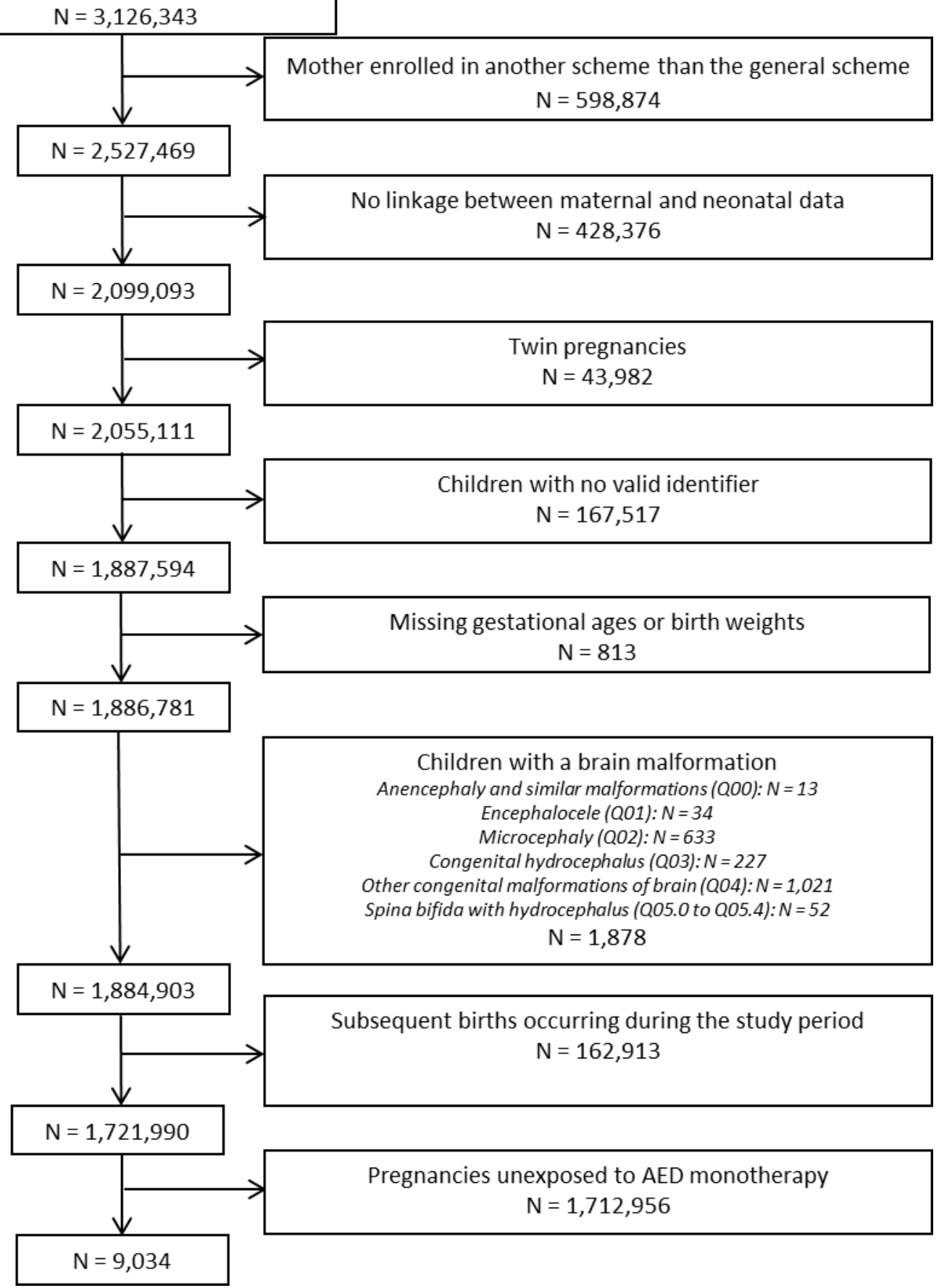

Figure 1 Inclusion flow chart. AED, antiepileptic drug.

and then by adding these daily doses overlapping with the pregnancy period. The number of days covered was defined as the sum of the 30-day periods of exposure corresponding to each refill minus the number of days of overlap between two consecutive refills. Only the days overlapping with the pregnancy period were taken into account.

\section{Outcomes}

Four outcomes were considered, based on the literature and on their availability in the French healthcare databases. The primary outcomes were hospitalisation or LTD for neurodevelopmental disorders (ICD-10 diagnosis codes F70-F98), and for two specific subcategories: pervasive developmental disorders (F84) and mental retardation (F70-F79). The secondary outcome was 'visits to a speech therapist', as a proxy for communication-related disorders.

\section{Follow-up}

Children were followed from birth until any of the predefined outcomes, loss to follow-up ( $>1$ year with no 
reimbursement), death from any cause or end of the study period defined as the 31 December 2016, whichever came first.

\section{Covariates}

Potential confounders related to the mother and considered in this study included sociodemographic covariates (maternal age at birth and eligibility for CMU-C), as well as comedications and comorbidities. Comedications included (1) preconception folic acid supplementation, defined as at least one dispensing between 1 month before pregnancy and 3 months after the start of pregnancy, (2) exposure to selective serotonin reuptake inhibitors (SSRIs) during pregnancy, (3) exposure to antipsychotics in the year before pregnancy, (4) a proxy for severity of mental disorders (the number of fifth-level ATC classes of psychiatric medications dispensed in the year before pregnancy). History of mental and behavioural disorders not related to alcohol or smoking, which was identified by using hospital discharge and LTD diagnoses, was also considered to be potential confounder. Alcohol intake and smoking were not directly available in the databases and proxies were calculated using modified versions of previously published algorithms. ${ }^{17}$ These proxies were constructed on the basis of hospital discharge diagnoses, LTD diagnoses and the child's hospital discharge diagnoses at birth. We also used specific drug reimbursements for alcohol intake and nicotine replacement therapy reimbursements for smoking. Potential confounders related to the child and considered in this study were gender, gestational age and birth weight.

All drugs and ICD-10 codes related to hospital discharge and LTD diagnoses used to define covariates are presented in online supplementary table 1 .

\section{Statistical analyses}

Baseline covariates were compared between pregnancies exposed to each AED studied and lamotrigine-exposed pregnancies using $\chi^{2}$ tests. Number of events, crude event rates and crude incidence rate ratios (IRRs) were calculated. Potential confounders were controlled for by performing inverse probability of treatment weighting (IPTW) analyses using the propensity score. These analyses were conducted separately according to the type of AED. Propensity scores were determined by using logistic regression models including the covariates listed above, with maternal age as a categorical variable $(\leq 25$ years, 25-30, 30-35 and $\geq 35$ ). Weights were trimmed at the 0.1 and 99.9 percentiles. Absolute standardised differences were calculated to assess the balance in baseline covariates before and after weighting. Groups were considered to be balanced when standardised differences were $<0.1{ }^{18}$ Cox models with robust sandwich estimates were used to calculate HRs and their $95 \%$ CIs.

As AEDs can be prescribed for a wide range of medical conditions other than epilepsy, a first sensitivity analysis in which the study population was restricted to women considered to be treated for epilepsy (see online supplementary table 1 for definition) was conducted. In this sensitivity analysis, HRs were further adjusted for hospitalisation (primary and related diagnoses only) for epilepsy during pregnancy. A second sensitivity analysis, requiring at least one dispensing during pregnancy to consider a woman to be exposed, was also conducted to account for possible misclassification of exposure at the beginning of pregnancy. In a third sensitivity analysis, two other propensity scores were calculated: one excluding proxies for alcohol intake and smoking and another one excluding gestational age and birth weight.

Data extraction and statistical analysis were performed by using SAS Enterprise Guide V.4.3 software (SAS Institute, Cary, North Carolina, USA). Graphics were performed by using R V.3.5 statistical software.

\section{RESULTS}

\section{Main analysis}

From a total of 1721990 births satisfying all inclusion and exclusion criteria, 9034 children were prenatally exposed to AED monotherapy (figure 1), $32(0.4 \%)$ of whom were censored at death and $1224(13.5 \%)$ were lost to follow-up. The median follow-up was 3.7 years (IOR, 2.7-4.7 years). Of these 9034 children, 2916 were exposed to lamotrigine, 1627 were exposed to pregabalin, 1246 were exposed to clonazepam, 991 were exposed to valproic acid, 621 were exposed to levetiracetam, 502 were exposed to carbamazepine, 477 were exposed to topiramate, 378 were exposed to gabapentin and 143 were exposed to oxcarbazepine (table 1). A total of 133 children were exposed to another AED monotherapy, including phenobarbital $(n=84)$, phenytoin $(n=13)$, lacosamide $(n=9)$, zonisamide $(n=8)$, ethosuximide $(\mathrm{n}=7)$ and vigabatrin $(\mathrm{n}=6)$. The median follow-up ranged from 3.3 to 4.0 years across all AEDs except clonazepam ( 4.8 years).

Table 1 also reports baseline patient characteristics according to the type of AED prior to IPTW, showing some significant differences between the various AEDs studied and lamotrigine. Before weighting, across all variables included in the propensity score and all AEDs studied, the absolute standardised differences ranged from 0.00 to 0.94 . After weighting, all standardised differences were $<0.1$, indicating a good balance between treatment groups (figure 2).

Table 2 presents the number of events, crude event rates, IRRs and adjusted HRs for the four outcomes and each of the AEDs studied. Compared with prenatal exposure to lamotrigine, prenatal exposures to all of the AEDs studied, excluding valproic acid, were not found to be associated with an increased risk of any of the four outcomes investigated. By contrast, valproic acid was associated with an increased risk of visits to a speech therapist $(\mathrm{HR}=1.5,95 \% \mathrm{CI}$ (1.1 to 1.9)) and neurodevelopmental disorders $(\mathrm{HR}=2.7$ (1.8 to 4.0$)$ ), particularly pervasive developmental disorders $(\mathrm{HR}=4.4$ (2.1 to 9.3) $)$ and mental retardation $(\mathrm{HR}=3.1$ (1.5 to 6.2$)$ ), compared with lamotrigine. 


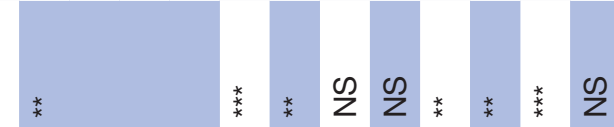

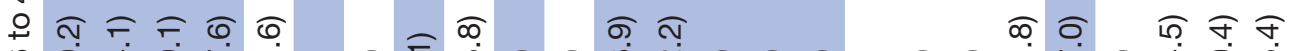

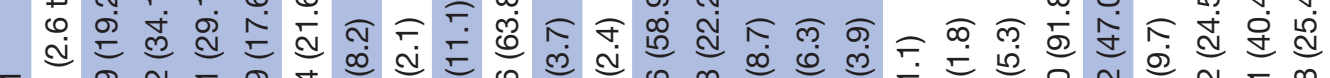
एٓ

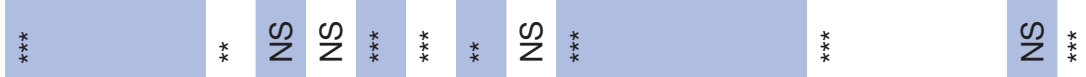

$\stackrel{\widehat{m}}{+\infty}$

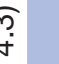

$\frac{\pi}{\frac{0}{\pi}}$

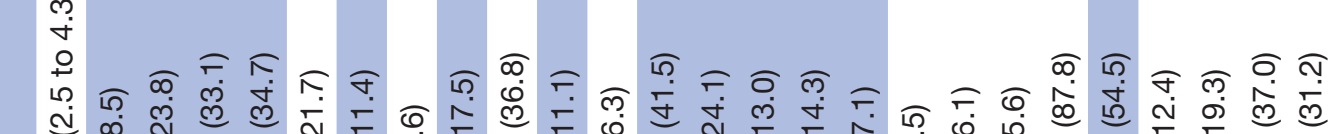

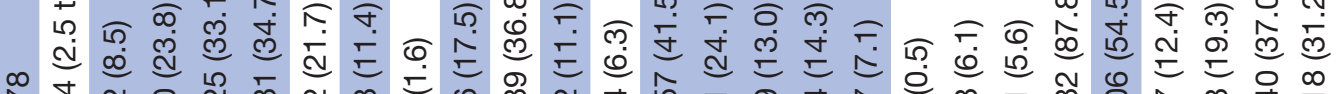
乔

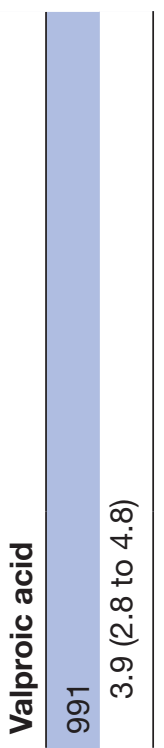

อิ

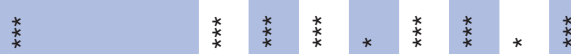

Q

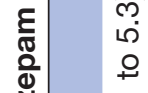

สุ เก क ल ल

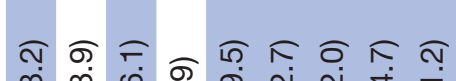

एक

0 过

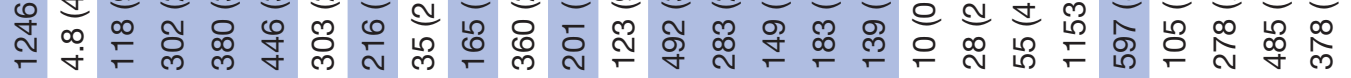

$\stackrel{0}{\stackrel{0}{c}}$

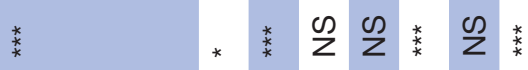

Q

C 2

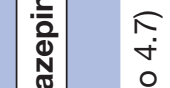

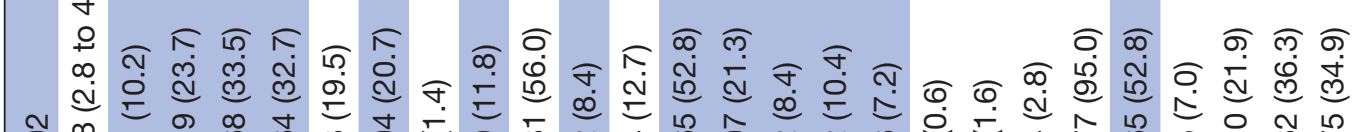
命

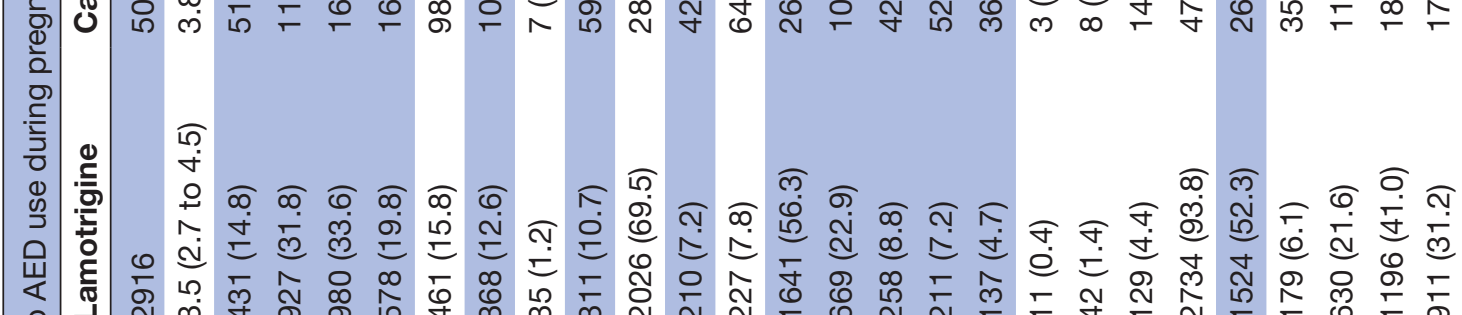
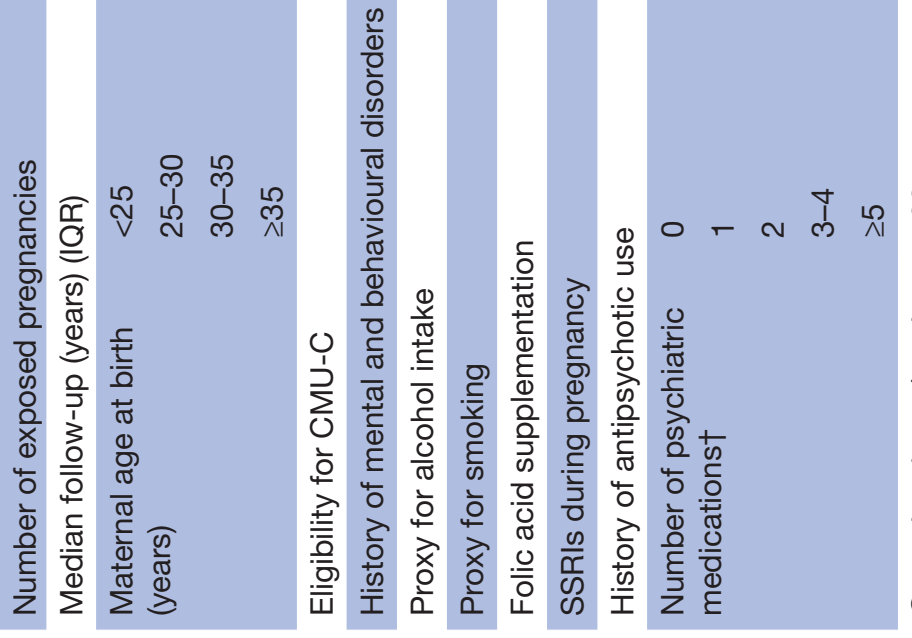

ᄂ

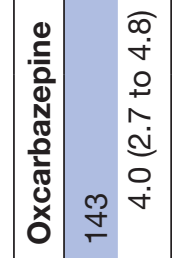




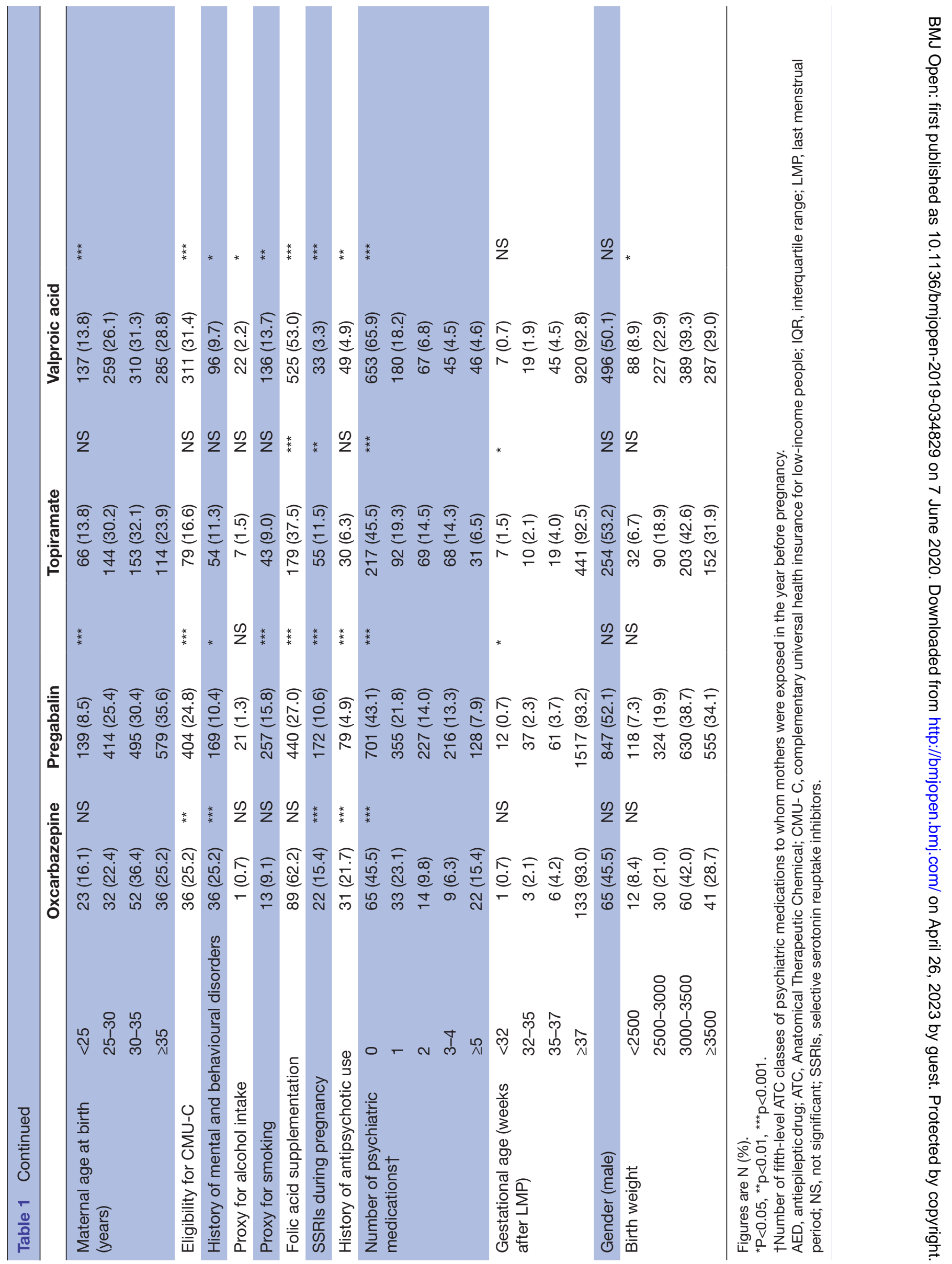




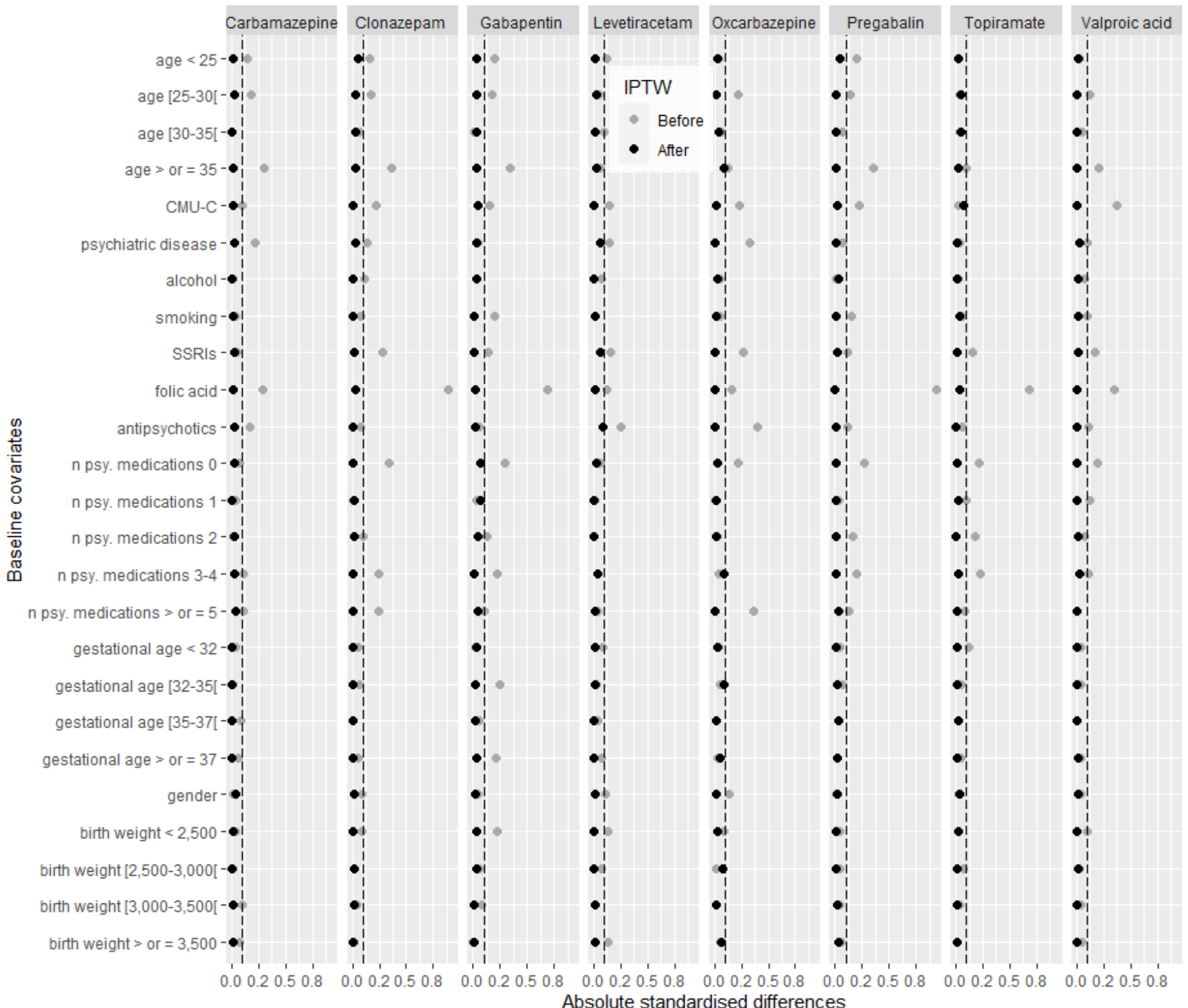

Figure 2 Differences in baseline covariates between children exposed to lamotrigine (reference group) and children exposed to the other AEDs studied before (grey dots) and after IPTW (black dots). AED, antiepileptic drug; IPTW, inverse probability of treatment weighting; CMU-C, complementary universal health insurance; $n$ psy. medications, number of Anatomical Therapeutic Chemical classes of psychiatric medications.

\section{Sensitivity analyses}

In the first sensitivity analysis restricting the study population to women considered to be treated for epilepsy, only results related to carbamazepine, levetiracetam and valproic acid are reported. The number of women considered to be treated for epilepsy and exposed to monotherapy with each of the other AEDs was $<100$. Baseline patient characteristics and absolute standardised differences are available in online supplementary table 3 and online supplementary figure 1 , respectively. Compared with lamotrigine, prenatal exposure to levetiracetam was not found to be associated with any of the four outcomes investigated and prenatal exposure to valproic acid was associated with all four outcomes, which is in line with the results of the main analysis. Results were different from the main analysis for the association between carbamazepine and the risk of visits to a speech therapist $(\mathrm{HR}=0.2,95 \%$ CI $(0.1$ to 0.7$)$ ) (table 3$)$.

The results of the second (see online supplementary figure 2 for standardised differences) and third sensitivity analyses were comparable to those of the main analysis (see online supplementary table 4 and online supplementary table 5 , respectively).

\section{Dose}

Baseline patient characteristics according to the dose of valproic acid dispensed during pregnancy are reported in online supplementary table 6 . A dose-response relationship was observed for the association between prenatal exposure to valproic acid and the risks of visits to a speech therapist (<700 mg: $\mathrm{HR}=0.6,95 \%$ CI ( 0.3 to 1.0$) ; 700-1500$ mg: $\mathrm{HR}=1.6$ (1.2 to 2.1 ); $\geq 1500 \mathrm{mg}$ : $\mathrm{HR}=2.6$ (1.7 to 4.0$)$ ) 
Table 2 Number of events, crude event rates (per 1000), crude IRRs and adjusted HRs for the four outcomes and each of the AEDs studied-main analysis

\begin{tabular}{|c|c|c|c|c|}
\hline & Events & Crude event rates & IRR & HR (95\% Cl) \\
\hline \multicolumn{5}{|c|}{ Neurodevelopmental disorders } \\
\hline Lamotrigine & 51 & 4.9 & & \\
\hline Carbamazepine & 13 & 7.0 & $1.4(0.8$ to 2.6$)$ & $1.2(0.6$ to 2.2$)$ \\
\hline Clonazepam & 28 & 5.1 & $1.0(0.6$ to 1.6$)$ & $0.6(0.4$ to 1.1$)$ \\
\hline Gabapentin & 4 & 3.1 & $0.6(0.2$ to 1.7$)$ & $0.4(0.1$ to 1.3$)$ \\
\hline Levetiracetam & 8 & 3.7 & $0.8(0.4$ to 1.6$)$ & 0.7 (0.3 to 1.5$)$ \\
\hline Oxcarbazepine & 3 & 5.6 & 1.1 (0.4 to 3.7$)$ & 0.8 (0.2 to 3.2$)$ \\
\hline Pregabalin & 28 & 5.0 & $1.0(0.6$ to 1.6$)$ & $0.6(0.4$ to 1.0$)$ \\
\hline Topiramate & 7 & 4.1 & $0.8(0.4$ to 1.8$)$ & $0.8(0.4$ to 1.9$)$ \\
\hline Valproic acid & 50 & 13.5 & $2.7(1.9$ to 4.0$)$ & 2.7 (1.8 to 4.0$)$ \\
\hline \multicolumn{5}{|c|}{ Pervasive developmental disorders } \\
\hline Lamotrigine & 11 & 1.1 & & \\
\hline Carbamazepine & 3 & 1.6 & $1.5(0.4$ to 5.4$)$ & $1.3(0.3$ to 5.0$)$ \\
\hline Clonazepam & 8 & 1.4 & $1.4(0.5$ to 3.4$)$ & $0.8(0.3$ to 2.1$)$ \\
\hline Gabapentin & 3 & 2.3 & $2.2(0.6$ to 7.9$)$ & $1.8(0.4$ to 7.0$)$ \\
\hline Levetiracetam & 4 & 1.8 & 1.8 (0.6 to 5.5$)$ & $1.6(0.5$ to 5.4$)$ \\
\hline Oxcarbazepine & 1 & 1.9 & $1.8(0.2$ to 13.6$)$ & 2.0 (0.3 to 13.8$)$ \\
\hline Pregabalin & 7 & 1.2 & $1.2(0.5$ to 3.1$)$ & $1.1(0.4$ to 3.0$)$ \\
\hline Topiramate & 1 & 0.6 & 0.6 (0.1 to 4.3$)$ & $0.3(0.0$ to 4.9$)$ \\
\hline Valproic acid & 17 & 4.5 & $4.3(2.0$ to 9.1$)$ & 4.4 (2.1 to 9.3$)$ \\
\hline \multicolumn{5}{|l|}{ Mental retardation } \\
\hline Lamotrigine & 15 & 1.4 & & \\
\hline Carbamazepine & 2 & 1.1 & 0.7 (0.2 to 3.2$)$ & 0.6 (0.1 to 2.9$)$ \\
\hline Clonazepam & 3 & 0.5 & 0.4 (0.1 to 1.3$)$ & 0.3 (0.1 to 1.2$)$ \\
\hline Gabapentin & 0 & 0.0 & N/A & $\mathrm{N} / \mathrm{A}$ \\
\hline Levetiracetam & 1 & 0.5 & $0.3(0.0$ to 2.4$)$ & $0.3(0.0$ to 2.5$)$ \\
\hline Oxcarbazepine & 0 & 0.0 & N/A & $\mathrm{N} / \mathrm{A}$ \\
\hline Pregabalin & 7 & 1.2 & $0.9(0.4$ to 2.1$)$ & $0.6(0.2$ to 1.8$)$ \\
\hline Topiramate & 2 & 1.2 & $0.8(0.2$ to 3.5$)$ & 0.5 (0.1 to 3.3$)$ \\
\hline Valproic acid & 15 & 4.0 & 2.8 (1.3 to 5.6$)$ & 3.1 (1.5 to 6.2$)$ \\
\hline \multicolumn{5}{|c|}{ Visits to a speech therapist } \\
\hline Lamotrigine & 157 & 15.2 & & \\
\hline Carbamazepine & 31 & 16.7 & 1.1 (0.8 to 1.6$)$ & 0.9 (0.6 to 1.4$)$ \\
\hline Clonazepam & 97 & 17.7 & $1.2(0.9$ to 1.5$)$ & $0.8(0.6$ to 1.0$)$ \\
\hline Gabapentin & 11 & 8.5 & 0.6 (0.3 to 1.0$)$ & 0.7 (0.4 to 1.2$)$ \\
\hline Levetiracetam & 22 & 10.2 & 0.7 (0.4 to 1.1$)$ & 0.7 (0.5 to 1.1$)$ \\
\hline Oxcarbazepine & 13 & 24.6 & 1.6 (0.9 to 2.9$)$ & $1.3(0.7$ to 2.5$)$ \\
\hline Pregabalin & 61 & 11.0 & $0.7(0.5$ to 1.0$)$ & 0.7 (0.5 to 1.0$)$ \\
\hline Topiramate & 33 & 19.4 & $1.3(0.9$ to 1.9$)$ & $1.2(0.8$ to 1.8$)$ \\
\hline Valproic acid & 93 & 25.1 & 1.7 (1.3 to 2.1$)$ & 1.5 (1.1 to 1.9$)$ \\
\hline
\end{tabular}

Lines marked in bold correspond to HRs for which the 95\% Cl does not include 1 .

AED, antiepileptic drug; Cl, confidence interval; HR, hazard ratio; IRR, incidence rate ratio; N/A, not applicable. 
Table 3 Number of events, crude event rates (per 1000), crude IRRs and adjusted HRs for the four outcomes and each of the AEDs studied-study population limited to women considered to be treated for epilepsy

\begin{tabular}{|c|c|c|c|c|c|}
\hline & Events & Crude event rates & IRR & $\mathrm{HR}^{*}(95 \% \mathrm{Cl})$ & $\mathrm{HR}+(95 \% \mathrm{Cl})$ \\
\hline \multicolumn{6}{|c|}{ Neurodevelopmental disorders } \\
\hline Lamotrigine & 32 & 4.2 & & & \\
\hline Carbamazepineł & 1 & 1.5 & 0.4 (0.0 to 2.7$)$ & 0.2 (0.0 to 2.7 ) & 0.2 (0.0 to 2.7 ) \\
\hline Levetiracetam & 8 & 3.7 & 0.9 (0.4 to 1.9$)$ & $0.8(0.4$ to 1.8$)$ & $0.8(0.4$ to 1.8$)$ \\
\hline Valproic acid & 50 & 13.5 & 3.2 (2.0 to 5.0$)$ & 3.2 (2.0 to 4.9 ) & 3.5 (2.3 to 5.4$)$ \\
\hline \multicolumn{6}{|c|}{ Pervasive developmental disorders } \\
\hline Lamotrigine & 7 & 0.9 & & & \\
\hline Carbamazepineł & 0 & 0.0 & $\mathrm{~N} / \mathrm{A}$ & $\mathrm{N} / \mathrm{A}$ & $\mathrm{N} / \mathrm{A}$ \\
\hline Levetiracetam & 4 & 1.8 & 2.0 (0.6 to 6.8$)$ & 1.8 (0.5 to 6.6$)$ & 1.8 (0.5 to 6.6$)$ \\
\hline Valproic acid & 17 & 4.5 & $4.9(2.0$ to 11.8$)$ & $4.9(2.0$ to 11.8$)$ & 4.7 (1.9 to 11.4 \\
\hline \multicolumn{6}{|l|}{ Mental retardation } \\
\hline Lamotrigine & 10 & 1.3 & & & \\
\hline Carbamazepineł & 0 & 0.0 & $\mathrm{~N} / \mathrm{A}$ & $\mathrm{N} / \mathrm{A}$ & $\mathrm{N} / \mathrm{A}$ \\
\hline Levetiracetam & 1 & 0.5 & 0.3 (0.0 to 2.7 ) & 0.4 (0.1 to 2.9$)$ & 0.4 (0.1 to 2.9$)$ \\
\hline Valproic acid & 15 & 4.0 & 3.0 (1.4 to 6.7$)$ & 3.1 (1.4 to 7.0$)$ & 4.0 (1.9 to 8.6$)$ \\
\hline \multicolumn{6}{|c|}{ Visits to a speech therapist } \\
\hline Lamotrigine & 122 & 16.2 & & & \\
\hline Carbamazepine $\ddagger$ & 4 & 6.1 & $0.4(0.1$ to 1.0$)$ & $0.2(0.1$ to 0.7$)$ & 0.2 (0.1 to 0.7$)$ \\
\hline Levetiracetam & 22 & 10.2 & 0.6 (0.4 to 1.0$)$ & 0.7 (0.4 to 1.1$)$ & 0.7 (0.4 to 1.1$)$ \\
\hline Valproic acid & 93 & 25.1 & $1.6(1.2$ to 2.0$)$ & 1.4 (1.1 to 1.8$)$ & 1.5 (1.1 to 1.9$)$ \\
\hline
\end{tabular}

Lines marked in bold correspond to HRs for which the $95 \% \mathrm{Cl}$ does not include 1 .

${ }^{*}$ Adjusted for all covariates except hospitalisation for epilepsy during pregnancy.

†Adjusted for all covariates including hospitalisation for epilepsy during pregnancy.

$\ddagger$ No possible adjustment for the proxy for alcohol.

AED, antiepileptic drug; $\mathrm{Cl}$, confidence interval; HR, hazard ratio; IRR, incidence rate ratio; N/A, not applicable.

and neurodevelopmental disorders $(<700 \mathrm{mg}: \mathrm{HR}=1.3$ (0.6 to 2.8 ); 700-1500 mg: HR=2.1 (1.3 to 3.5$) ; \geq 1500 \mathrm{mg}$ : $\mathrm{HR}=7.0$ (4.3 to 11.5$)$ ), including pervasive developmental disorders $(<700 \mathrm{mg}: \mathrm{HR}=2.2$ (0.5 to 8.5$)$; 700-1500 mg: $\mathrm{HR}=2.7$ (1.0 to 7.1$) ; \geq 1500 \mathrm{mg}: \mathrm{HR}=14.7$ (6.2 to 34.7$)$ ). The highest HR of mental retardation was also observed for the highest mean daily dose $(\geq 1500 \mathrm{mg}$ : HR=7.3 (3.0 to 17.7)). Comparable results were observed when the study population was limited to women considered to be treated for epilepsy (table 4).

\section{DISCUSSION}

\section{Main findings}

In this nationwide observational study based on the French healthcare databases, prenatal exposures to levetiracetam, pregabalin, oxcarbazepine, topiramate, gabapentin, clonazepam and carbamazepine were not associated with an increased risk of any of the early neurodevelopmental outcomes investigated compared with lamotrigine. The decreased risk of visits to a speech therapist observed with carbamazepine when the population was restricted to women with epilepsy may be a chance finding. This association is based on only four children and carbamazepine was associated with reduced verbal abilities in one study. ${ }^{19}$ Prenatal exposure to valproic acid was found to be associated with increased risks of all neurodevelopmental outcomes investigated compared with lamotrigine, ranging from pervasive mental disorders to possible communication-related disorders. A dose-response relationship was observed for most of these outcomes.

\section{Comparison with previous studies}

Prenatal exposure to lamotrigine has been mostly shown not to be associated with poorer neurodevelopmental outcomes, although limited data are available, with no dose-response relationship. ${ }^{6}$ Only two studies have shown that prenatal exposure to lamotrigine was associated with impaired specific cognitive skills ${ }^{20}$ and parental concerns about autistic traits and sentence skills. ${ }^{21}$

The results of the present study concerning valproic acid, carbamazepine, levetiracetam and topiramate are consistent with those of previous studies. Prenatal exposure to valproic acid has been associated with poorer cognitive outcomes ${ }^{1922-24}$ and particularly poorer executive functions and memory abilities ${ }^{23}$ compared with lamotrigine. Children prenatally exposed to valproic acid 


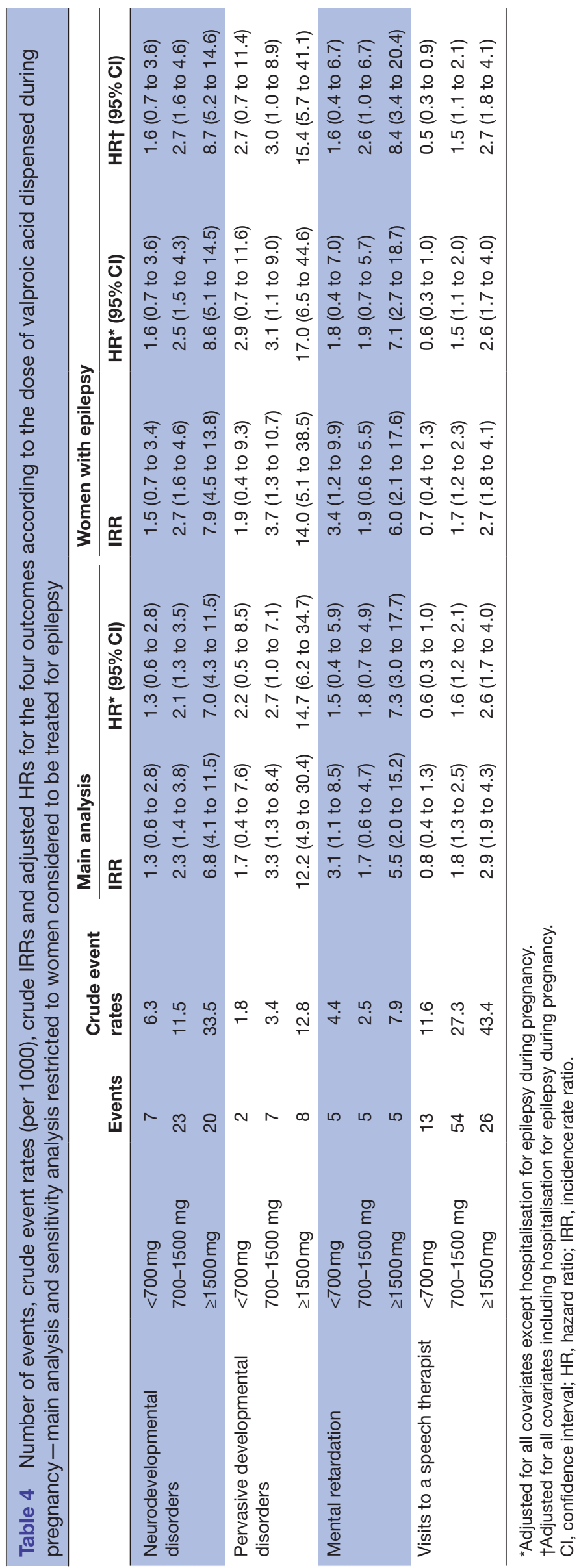


also show impaired adaptive behaviour ${ }^{25} 26$ and school performance ${ }^{27}$ compared with lamotrigine-exposed children. Impaired language skills ${ }^{28}$ and autism spectrum disorder have been reported to be more frequent among children exposed to valproic acid than among unexposed children. ${ }^{2930}$ A dose-response relationship has also been observed for most of these outcomes. ${ }^{19} 2223252628$

Few studies have directly compared prenatal exposure with carbamazepine versus lamotrigine and these studies did not find any differences in terms of cognitive development ${ }^{22}{ }^{31}$ and adaptive behaviour. ${ }^{26}$ Data concerning levetiracetam and topiramate are more limited and no direct comparison with lamotrigine has been published. No increased risk of neurodevelopmental outcomes was found among levetiracetam-exposed children compared with unexposed children ${ }^{32-34}$ and, although prenatal exposure to topiramate was associated with poorer neurodevelopmental outcomes in one study, ${ }^{35}$ this association was not confirmed by a larger study. ${ }^{33}$ Little or no information is available concerning the other AEDs, particularly clonazepam, oxcarbazepine, gabapentin and pregabalin. 293336

\section{Strengths}

This nationwide cohort study based on the French healthcare database is the largest study to date to assess the association between AED exposure during pregnancy and neurodevelopmental outcomes in the offspring. This study investigated a wide range of AEDs, including some drugs for which little or no information is available in the literature. The strengths of this study also include the use of propensity score methods to mitigate confounding, as well as the advantages of the French healthcare databases, such as the independence between ascertainment of medication and outcomes, the absence of recall bias, and the possibility to study the dose-response relationship, which is a key concept in terms of teratogenicity. ${ }^{33}$

\section{Limitations}

Exposure misclassification constitutes a first limitation. Exposure assessment was based on pharmacy claims, which do not indicate whether the medication is actually taken. Exposure misclassification is more likely for AED classes that are often discontinued before conception. ${ }^{37}$ However, the results of the sensitivity analysis regarding exposure measurement suggest that this bias is likely minimal.

Although we used an active-comparator design, residual confounding by unmeasured or insufficiently wellmeasured covariates cannot be excluded. For instance, maternal education and IQ were not available in the databases. Data related to the father could not be linked to the child, not allowing any adjustment for paternal characteristics. Lifestyle factors such as alcohol intake and smoking could also not be exhaustively assessed.

As some AEDs can be prescribed to treat conditions other than epilepsy, the analyses were replicated in a population restricted to women considered to be treated for epilepsy, providing comparable results to those of the main analysis. However, the indication for which an AED is prescribed was not directly available in the databases. We therefore used LTD diagnoses, hospital diagnoses and specific drug reimbursements to identify women with epilepsy. Some studies, ${ }^{34} 3839$ unlike other studies, ${ }^{19}$ 22-24 have also reported an association between the severity of maternal epilepsy, particularly seizure type and frequency, and poorer developmental outcomes in the child. As this confounder was not available in the databases, we considered at least one admission to hospital for epilepsy during pregnancy to be a proxy for epilepsy severity. Similar results were observed whether or not this proxy was included in the propensity score.

Outcome misclassification also cannot be ruled out, as the diagnosis codes used to identify neurodevelopmental outcomes have not been externally validated. However, the PMSI database is used for planning and funding purposes and is subject to coding quality control, and LTD registration, which is requested by the patient's general practitioner, must be validated by a medical consultant of the beneficiary's health insurance scheme. In addition, as hospital discharge and LTD diagnoses were used to define outcomes, children not reaching diagnostic thresholds but still having some evidence of impairment were not considered to have experienced the outcomes of interest. ${ }^{40}$ Moreover, some of the outcomes studied may not have been exhaustively assessed, as data related to a large share of medical and social welfare services are not available in the French healthcare databases. Results related to visits to a speech therapist must also be interpreted with caution, as a visit to a speech therapist does not necessarily imply pathology. Speech therapists can also be consulted for various medical reasons, and access to speech therapists is associated with socioeconomic status. ${ }^{41}$ Furthermore, because the study outcomes were limited to three subtypes of neurodevelopmental disorders (pervasive developmental disorders, mental retardation and visits to a speech therapist as a proxy for communication-related disorders), no conclusion can be drawn concerning all of the other subtypes of neurodevelopmental disorders.

The short follow-up period constitutes another limitation: the median and maximum lengths of follow-up were 3.7 and 6 years, respectively. In particular, a diagnosis of autism spectrum disorders is considered to be stable at age $2^{42}$ and is made at an average age of 3 years and 5 months in France, ${ }^{43}$ but speech therapy is more frequent among school-aged children. Therefore, only early diagnoses, probably corresponding to more severe disorders, were identified. This is especially true for mental retardation: although severe and profound mental retardation can be diagnosed before 3 years of age, moderate mental retardation cannot be diagnosed before 4 or 5 years of age. ${ }^{44}$ Further assessment of children at older developmental stages would be useful to study a broader range of neurodevelopmental disorders. Except clonazepam for which French health authorities took measures to limit off-label 
use in November $2011,{ }^{45}$ lengths of follow-up were quite comparable across all AEDs. Children exposed to secondgeneration AEDs had only slightly shorter follow-ups than children exposed to first-generation AEDs, which should not have influenced the results of this study.

\section{CONCLUSION}

Despite the limitations inherent to healthcare claims databases, this study, based on 9034 exposed children, confirms that valproic acid is associated with an increased risk of various neurodevelopmental outcomes compared with lamotrigine, with a dose-response relationship, while no association was observed for the other AEDs including carbamazepine, levetiracetam or topiramate. However, this study needs to be replicated with a longer follow-up period.

\section{Author affiliations}

${ }^{1}$ Department of Public Health Studies, French National Health Insurance (CNAM), Paris, France

${ }^{2}$ Apemac, EA 4360, Université de Lorraine, Université Paris-Descartes, Nancy, France

${ }^{3}$ Department of Epidemiology of Health Products, French National Agency for Medicines and Health Products Safety, Saint-Denis, France

${ }^{4}$ Assistance Publique-Hôpitaux de Paris, Hôpital Bicêtre, Unité de Rééducation Neurologique Infantile, Bicêtre, France

${ }^{5} \mathrm{CESP}$, Faculté de médecine-Université Paris-Sud, Faculté de médecine-UVSQ, INSERM, Université Paris-Saclay, Villejuif, France

${ }^{6}$ Laboratoire de Sciences Cognitives et Psycholinguistique, Ecole Normale

Supérieure, EHESS, CNRS, PSL University, Paris, France

${ }^{7}$ Department of Child and Adolescent Psychiatry, Robert Debré Hospital, APHP, Paris, France

${ }^{8}$ INSERM UMR 1141, Paris Diderot University, Paris, France

${ }^{9}$ Versailles Saint-Quentin-en-Yvelines University, Versailles, France

${ }^{10}$ Biostatistics and Epidemiology Unit, Hôtel-Dieu Hospital, Assistance Publique-

Hôpitaux de Paris, and Paris Descartes University, Paris, France

Acknowledgements The authors would like to thank François Alla and Géric Maura for assistance in revising the manuscript for intellectual content and Anthony Saul, medical translator, for assistance in editing the manuscript.

Contributors P-OB, SM, AW, MZ, JC and RD-S contributed to the conception and design of the study. $\mathrm{P}-\mathrm{OB}$ and $\mathrm{SM}$ contributed to the acquisition of data. $\mathrm{P}-\mathrm{OB}$ conducted the statistical analyses. P-OB, SM, AW, YM, HP, FR, MZ, JC and RD-S contributed to the interpretation of data. P-OB drafted the manuscript. AW, YM, HP, $F R, M Z, J C$ and RD-S revised the manuscript for important intellectual content. AW, $\mathrm{MZ}, \mathrm{JC}$ and RD-S coordinated and supervised the study.

Funding $\mathrm{P}-\mathrm{OB}$ and $\mathrm{AW}$ are employees of the French National Health Insurance (CNAM). SM, MZ and RD-S are employees of the French National Agency for Medicines and Health Products Safety (ANSM), YM and JC work at Assistance Publique-Hôpitaux de Paris. None of these authors received any funding. All work by HP and FR is supported by Agence Nationale de la Recherche (grants ANR-17EURE-0017 and ANR-10-IDEX-0001-02).

Competing interests None declared.

Patient and public involvement Patients and/or the public were not involved in the design, conduct, reporting or dissemination plans of this research.

Patient consent for publication Not required.

Ethics approval This observational cohort study based on the French healthcare databases was approved by the French Data Protection Agency (CNIL, approval ID: DE-2015-165).

Provenance and peer review Not commissioned; externally peer reviewed.

Data availability statement No data are available. Permanent access to the French health care databases is automatically granted to certain government agencies, public institutions and public service authorities. Temporary access for studies and research is possible on request from the National Health Data Institute (INDS).

Open access This is an open access article distributed in accordance with the Creative Commons Attribution Non Commercial (CC BY-NC 4.0) license, which permits others to distribute, remix, adapt, build upon this work non-commercially, and license their derivative works on different terms, provided the original work is properly cited, appropriate credit is given, any changes made indicated, and the use is non-commercial. See: http://creativecommons.org/licenses/by-nc/4.0/.

\section{ORCID iDs}

Pierre-Olivier Blotière http://orcid.org/0000-0002-6857-6781

Rosemary Dray-Spira http://orcid.org/0000-0001-7646-3667

\section{REFERENCES}

1 Spina E, Perugi G. Antiepileptic drugs: indications other than epilepsy. Epileptic Disord Int Epilepsy J Videotape 2004;6:57-75.

2 Charlton R, Garne E, Wang H, et al. Antiepileptic drug prescribing before, during and after pregnancy: a study in seven European regions. Pharmacoepidemiol Drug Saf 2015;24:1144-54.

3 Blotière $\mathrm{P}-\mathrm{O}$, Weill A, Dalichampt $\mathrm{M}$, et al. Development of an algorithm to identify pregnancy episodes and related outcomes in health care claims databases: an application to antiepileptic drug use in 4.9 million pregnant women in France. Pharmacoepidemiol Drug Saf 2018;27:763-70.

4 Bobo WV, Davis RL, Toh S, et al. Trends in the use of antiepileptic drugs among pregnant women in the US, 2001-2007: a medication exposure in pregnancy risk evaluation program study. Paediatr Perinat Epidemiol 2012;26:578-88.

5 Hill DS, Wlodarczyk BJ, Palacios AM, et al. Teratogenic effects of antiepileptic drugs. Expert Rev Neurother 2010;10:943-59.

6 Bromley RL, Baker GA. Fetal antiepileptic drug exposure and cognitive outcomes. Seizure 2017;44:225-31.

7 US Food and Drug administration. FDA drug safety communication: children born to mothers who took valproate products while pregnant may have impaired cognitive development, 2011. Available: https:// www.fda.gov/Drugs/DrugSafety/ucm261543.htm [Accessed 14 Jan 2019].

8 US Food and Drug administration. FDA drug safety communication: valproate anti-seizure products Contraindicated for migraine prevention in pregnant women due to decreased IQ scores in exposed children, 2013. Available: https://www.fda.gov/drugs/ drugsafety/ucm350684.htm [Accessed 6 Aug 2018].

9 European Medicines Agency. CMDh agrees to strengthen warnings on the use of valproate medicines in women and girls, 2014. Available: https://www.ema.europa.eu/en/medicines/human/referrals/ valproate-related-substances [Accessed 14 Jan 2019].

10 European Medicines Agency. New measures to avoid valproate exposure in pregnancy endorsed, 2018. Available: https://www. ema.europa.eu/en/medicines/human/referrals/valproate-relatedsubstances-0 [Accessed 14 Jan 2019].

11 Tuppin P, Rudant J, Constantinou P, et al. Value of a national administrative database to guide public decisions: from the Système national d'information interrégimes de I'Assurance Maladie (SNIIRAM) to the Système national des données de santé (SNDS) in France. Rev Epidemiol Sante Publique 2017;65(Suppl 4):S149-67.

12 Fonds CMU-C. Présentation de la CMU-C. Available: https://www. cmu.fr/cmu-complementaire.php [Accessed 12 Jul 2018].

13 Raguideau F, Mezzarobba M, Zureik M, et al. Compliance with pregnancy prevention plan recommendations in 8672 French women of childbearing potential exposed to acitretin. Pharmacoepidemiol Drug Saf 2015;24:526-33.

14 Billionnet C, Mitanchez D, Weill A, et al. Gestational diabetes and adverse perinatal outcomes from 716,152 births in France in 2012. Diabetologia 2017;60:636-44.

15 Blotière P-O, Raguideau F, Weill A, et al. Risks of 23 specific malformations associated with prenatal exposure to 10 antiepileptic drugs. Neurology 2019;93:e167-80.

16 Lund JL, Richardson DB, Stürmer T. The active comparator, new user study design in pharmacoepidemiology: historical foundations and contemporary application. Curr Epidemiol Rep 2015;2:221-8.

17 Maura G, Billionnet C, Alla F, et al. Comparison of treatment persistence with dabigatran or rivaroxaban versus vitamin $\mathrm{K}$ antagonist oral anticoagulants in atrial fibrillation patients: a competing risk analysis in the French National health care databases. Pharmacotherapy 2018;38:6-18. 
18 Austin PC, Stuart EA. Moving towards best practice when using inverse probability of treatment weighting (IPTW) using the propensity score to estimate causal treatment effects in observational studies. Stat Med 2015;34:3661-79.

19 Baker GA, Bromley RL, Briggs M, et al. Iq at 6 years after in utero exposure to antiepileptic drugs: a controlled cohort study. Neurology 2015;84:382-90.

20 Rihtman T, Parush S, Ornoy A. Developmental outcomes at preschool age after fetal exposure to valproic acid and lamotrigine: cognitive, motor, sensory and behavioral function. Reprod Toxicol 2013;41:115-25.

21 Veiby G, Daltveit AK, Schjølberg S, et al. Exposure to antiepileptic drugs in utero and child development: a prospective populationbased study. Epilepsia 2013;54:1462-72.

22 Bromley RL, Mawer G, Love J, et al. Early cognitive development in children born to women with epilepsy: a prospective report. Epilepsia 2010;51:2058-65.

23 Meador KJ, Baker GA, Browning N, et al. Fetal antiepileptic drug exposure and cognitive outcomes at age 6 years (NEAD study): a prospective observational study. Lancet Neurol 2013;12:244-52.

24 Meador KJ, Baker GA, Browning N, et al. Cognitive function at 3 years of age after fetal exposure to antiepileptic drugs. $N$ Engl J Med 2009;360:1597-605

25 Cohen MJ, Meador KJ, Browning N, et al. Fetal antiepileptic drug exposure: adaptive and emotional/behavioral functioning at age 6years. Epilepsy Behav 2013;29:308-15.

26 Deshmukh U, Adams J, Macklin EA, et al. Behavioral outcomes in children exposed prenatally to lamotrigine, valproate, or carbamazepine. Neurotoxicol Teratol 2016;54:5-14.

27 Elkjær LS, Bech BH, Sun Y, et al. Association between prenatal valproate exposure and performance on standardized language and mathematics tests in school-aged children. JAMA Neurol 2018;75:663

28 Nadebaum C, Anderson VA, Vajda F, et al. Language skills of schoolaged children prenatally exposed to antiepileptic drugs. Neurology 2011;76:719-26.

29 Christensen J, Grønborg TK, Sørensen MJ, et al. Prenatal valproate exposure and risk of autism spectrum disorders and childhood autism. JAMA 2013;309:1696-703.

30 Bromley RL, Mawer GE, Briggs M, et al. The prevalence of neurodevelopmental disorders in children prenatally exposed to antiepileptic drugs. J Neurol Neurosurg Psychiatry 2013;84:637-43.

31 Bromley R, Weston J, Adab N, et al. Treatment for epilepsy in pregnancy: neurodevelopmental outcomes in the child. Cochrane Database Syst Rev 2014:CD010236.
32 Shallcross R, Bromley RL, Irwin B, et al. Child development following in utero exposure: levetiracetam vs sodium valproate. Neurology 2011;76:383-9.

33 Bromley RL, Calderbank R, Cheyne CP, et al. Cognition in school-age children exposed to levetiracetam, topiramate, or sodium valproate. Neurology 2016;87:1943-53.

34 Shallcross R, Bromley RL, Cheyne CP, et al. In utero exposure to levetiracetam vs valproate: development and language at 3 years of age. Neurology 2014;82:213-21.

35 Rihtman T, Parush S, Ornoy A. Preliminary findings of the developmental effects of in utero exposure to topiramate. Reprod Toxicol 2012;34:308-11.

36 Miškov S, Gjergja Juraški R, Mikula I, et al. The Croatian model of integrative prospective management of epilepsy and pregnancy. Acta Clin Croat 2016:55:535-48.

37 Grzeskowiak LE, Gilbert AL, Morrison JL. Exposed or not exposed? Exploring exposure classification in studies using administrative data to investigate outcomes following medication use during pregnancy. Eur J Clin Pharmacol 2012;68:459-67.

38 Adab N, Kini U, Vinten J, et al. The longer term outcome of children born to mothers with epilepsy. J Neurol Neurosurg Psychiatry 2004;75:1575-83.

39 Vinten J, Adab N, Kini U, et al. Neuropsychological effects of exposure to anticonvulsant medication in utero. Neurology 2005;64:949-54.

40 Thapar A, Cooper M, Rutter M. Neurodevelopmental disorders. Lancet Psychiatry 2017;4:339-46.

41 Morgan PL, Hammer CS, Farkas G, et al. Who receives Speech/ Language services by 5 years of age in the United States? Am J Speech Lang Pathol 2016;25:183-99.

42 Kleinman JM, Ventola PE, Pandey J, et al. Diagnostic stability in very young children with autism spectrum disorders. J Autism Dev Disord 2008;38:606-15

43 Third Autism Plan [Troisième Plan Autisme] (2013-2017). Available: https://www.cnsa.fr/documentation/plan-autisme2013.pdf

44 French National Institute for Health and Medical Research (INSERM). Synthesis of the collective experience "Intellectual disabilities" [Synthèse de l'expertise collective "Déficiences intellectuelles"], 2016. Available: https://www.inserm.fr/information-en-sante/ expertises-collectives/deficiences-intellectuelles

45 Agence nationale de sécurité du médicament et des produits de santé (ANSM). Clonazepam: modification of the conditions of prescription or dispensing [Rivotril ${ }^{\circ}$ (clonazépam) : Modification des conditions de prescription et de délivrance], 2011. Available: https:// ansm.sante.fr/S-informer/Points-d-information-Points-d-information/ Rivotril-R-clonazepam-Modification-des-conditions-de-prescriptionet-de-delivrance-Point-d-information [Accessed 23 Apr 2019]. 